\title{
Verso un sapere sul sacro: dal misticismo spagnolo alla ragione poetica
}

\author{
Towards a Knowledge of the Sacred: \\ From Spanish Mysticism to Poetic Reason
}

Veronica Tartabini

Universidad Autónoma de Madrid veronica.tartabini@uam.es

Orcid: 0000-0002-5346-4429

DOI: https://doi.org/10.15366/bp2020.25.012

Bajo Palabra. II Época. No25. Pgs: 251-272 


\section{Resumen}

San Juan de la Cruz y Teresa de Ávila ejercieron su influencia en las reflexiones de una de las protagonistas de la filosofía española del siglo XX: María Zambrano.

En 1939, dando los primeros pasos de su exilio en México, la filósofa escribió sobre el gran místico castellano heredando su crítica hacia una concepción del hombre que pueda prescindir del alma y de las "entrañas" de la existencia, del amor a la vida y sus diferencias, de la unión de la filosofía y la poesía. En su madurez, en Roma, la pensadora malagueña escribe citando a Santa Teresa y su visión de la subjetividad humana, heredando sus enseñanzas para comprender al ser humano en su constante búsqueda de unión con lo sagrado. Es una amplia tradición de pensamiento, la que sitúa a la alumna de Ortega en la estela de los grandes místicos españoles del siglo XVI.

Palabras clave: María Zambrano, Mistica, Santa Teresa de Ávila, San Juan de la Cruz, Sagrado, Filosofía, Poesia, Razón Poética.

\section{Abstract}

St. John of the Cross and Saint Teresa of Avila exercised their influence on the reflections of one of the protagonists of 20th century Spanish philosophy: María Zambrano.

In 1939, taking the first steps towards her exile in Mexico, the philosopher writes on the great Castilian mystic inheriting the critique of a conception of man that can set aside the soul and the "bowels" of existence, love for life and its differences, the union of philosophy and poetry. In her maturity, in Rome, the thinker of Malaga writes quoting Saint Teresa and her vision of human subjectivity, inheriting her teachings to understand the human being in his constant search for union with the sacred. It is a broad tradition of thought that places Ortega's pupil in the wake of the sixteenth century great Spanish mystics.

Keywords: Maria Zambrano, Mysticism, Saint Teresa of Avila, Saint John of the Cross, Sacred, Philosophy, Poetry, Poetic Reason. 
Agli esiliati spagnoli che nel 1939, varcando una frontiera, aprirono una ferita dilaniante nei loro cuori e nell'anima del mondo.

Una fessura sanguinante nelle pieghe della nostra storia, in cui è necessario entrare per guardare negli occhi il nostro presente ed affrontarlo.

Alle menti come Doña María, che hanno impugnato le asperità dell'esistenza e le hanno rese lo sprone per conoscersi a fondo, unendo le viscere della vita al divino.

\section{Introduzione}

[...] la personalità più eminente [...] era María Zambrano, una gentile figura di filosofa con tratti, intensamente poetici, di profetessa, che la facevano rientrare nella grande tradizione mistica spagnola: salvo che era stata, e rimaneva, politicamente appassionatissima.

Elena Croce, Due città.

\section{L'imperatore Carlo V d'Asburgo, o se si preferisce, Carlo I di Spagna custo- diva nelle sue stanze del monastero di Yuste un dipinto ${ }^{1}$ di Tiziano. Il sovrano}

${ }^{1}$ La Gloria, chiamata anche Trinità o Paradiso, di Tiziano è una rappresentazione allegorica del cattolicesimo. L'opera simboleggia la profonda devozione per la Trinità della famiglia imperiale. Prima ubicazione del dipinto fu il monastero di Yuste, presso la stanza dove Carlo V d'Asburgo trascorse i suoi ultimi giorni di vita. Sua seconda destinazione fu l'Aula Morale del Monastero dell'Escorial fino a quando, nel 1837, comparve nella collezione del Museo del Prado. Notiamo alla sommità della struttura pittorica, i grandi protagonisti del cattolicesimo: la Trinità (Dio Padre, Figlio e Spirito Santo nelle sembianze di una colomba), accompagnata dalla Vergine Maria - la figura femminile che indossa l'abito blu- e San Giovanni Battista. San Giovanni e la Vergine godono di un sito privilegiato in quanto intercessori per eccellenza tra il piano divino e gli uomini. Colti nel momento in cui discendono dalle nuvole dorate in Gloria, distinguiamo altri personaggi dell'Antico e del Nuovo Testamento. Ad esempio Mosè, con le Tavole dei Dieci comandamenti; Noè che alza una piccola Arca, in cui cerca equilibrio una colomba con un ramo nel becco e Re Davide, alle prese con un'arpa. Tra tali figure adoranti la Trinità, si scorge di schiena, un'immagine femminile con indumenti verdi che ha prodotto le più svariate interpretazioni: è stata definita come Maria Maddalena, come la Sibilla Eritrea (che profetizzò sul Giudizio Universale), come Giuditta, Rachele o, probabilmente la Chiesa cattolica. Sulla destra, è riconoscibile Carlo I di Spagna, al lato della sua corona imperiale, seguito dai membri della famiglia reale. Il sovrano è accompagnato dalla sua consorte, Isabella del Portogallo; suo figlio Filippo II, destinato a succedergli al trono; sua figlia Giovanna d'Asburgo; Maria, Regina consorte d'Ungheria, ed infine Leonor, Regina di Francia e del Portogallo.

Poco più in basso nella composizione, un uomo con la barba è stato identificato con lo stesso Tiziano. L'Evangelista Giovanni (riconoscibile grazie all'aquila, suo simbolo), sorregge un foglio firmato dal pittore italiano. La 
aveva commissionato il lavoro al grande pittore veneziano per poter ammirare, ai piedi del suo capezzale, la sua visione dell'ingresso nell'al di là. Si tratta della prima opera che ha avviato la realizzazione dell'intera collezione del Museo del Prado. Dunque, nell'epoca dei grandi mistici iberici, l'imperatore del Sacro Romano Impero Germanico, figlio di Giovanna la Pazza e fermo difensore del cristianesimo, scelse l'arte italiana per far raffigurare la sua esperienza spirituale, la stessa che tentava di diffondere nei suoi possedimenti tanto estesi da "non vedere mai tramontare il sole". Italia e Spagna: potere, religione, arte e legame mistico nel corso della modernità. Un legame che aveva ben presente anche María Zambrano, quando a secoli di distanza dalle pennellate poste sulla tela del Vecellio, compone i tratti del suo pensiero filosofico seguendo le linee guida di San Giovanni della Croce e Santa Teresa d'Ávila ${ }^{2}$. L'autrice andalusa rifletterà sul suo tempo, scriverà del suo tempo tra Spagna e Italia, esattamente sulle orme di quel Rinascimento italiano che tanto era riuscito a penetrare nel tessuto del Rinascimento della sua terra di origine. L'obiettivo delle riflessioni che qui cercheremo di organizzare è un tentativo di difesa della seguente tesi: è lecito parlare della filosofia zambraniana, quale pensiero dalle radici profondamente mistiche ${ }^{3}$. Tessere il filo, ricostruire l'eredità che unisce la filosofa di Malaga ai due principali esponenti della mistica spagnola del xvi secolo prima citati ${ }^{4}$,

cornice della scena è un tripudio di cori celesti ed angeli. L'olio su tela è stato ispirato dalla lettura de "La città di Dio" di Sant'Agostino d' Ippona, i cui passi trattano anche della gloria riservata ai beati. Il titolo definitivo de La Gloria fu scelto da José de Sigüenza nel 1601. È consigliata l'analisi del dipinto, supportata dal materiale grafico riportato alla fine del presente testo.

${ }^{2}$ La letteratura bibliografica sui due grandi mistici è vasta. Consigliamo tra gli ultimi studi di Rosa Rossi: Rossi, R., Juan de la Cruz. Silencio y creatividad, Traducción de Juan-Ramón Capella, Madrid, Minima Trotta, 2010 e Rossi, R., Teresa de Ávila. Biografía de una escritora, Madrid, Editorial Trotta, 2015.

${ }^{3}$ Una versione di questo articolo è stata presentata in occasione del Congresso Internazionale: "María Zambrano: mujer y pensadora. Una visión italo-española", che ha avuto luogo il 14 e il 15 febbraio 2019, presso la Reale Accademia di Spagna a Roma. Nella Real Academia de España en Roma, parlare di mistica risulta essere quanto mai appropriato per la storia che lega il Tempietto del Bramante alla morte di San Pietro, alle estasi di Amadeo Menes de Silva e per le lunette dedicate al ciclo francescano. Difatti, tale prestigiosa sede nasce in un luogo in cui la tradizione vuole che sia morto San Pietro. In onore dell'importanza sacrale del sito, Sisto IV nel 1472 concede al confessore Amadeo Menes de Silva la zona dove attualmente si trova il Tempietto. Le sue estasi avvenute nel luogo sacro, lo spingono ad incentivare l'avvio dei lavori finanziati prima dal sovrano di Francia, Luigi XI, e poi dai Re Cattolici di Spagna. Non possiamo non far cenno, inoltre, ai trenta affreschi che occupano le trenta lunette del perimetro esterno del Chiostro, i quali insieme a quelli del vicino Claustro del Tempietto, costituiscono uno dei cicli più estesi inerenti alla storia di San Francesco e il suo ordine. Questi affreschi furono opera del "Pomarancio" o Nicolò Cirignani (1587-1788) e furono finanziati dal mecenate Costanzo Boccafuoco. Parlando di tempi più vicini ai nostri giorni, ricordiamo la mostra "Estasi mistica e pienezza creativa. Omaggio a Santa Teresa d'Ávila" dell'artista Omar Galliani, che dal 22 aprile al 31 maggio del 2016 ha trovato nel Tempietto presso San Pietro in Montorio, la scenografia perfetta per un'ulteriore occasione di legame tra misticismo spagnolo e contesto culturale italiano.

${ }^{4}$ Anche se San Giovanni della Croce è cronologicamente successivo a Santa Teresa, partiamo da un confronto con il mistico andaluso perché con lui possiamo analizzare tematiche più generali, per poi approfondire aspetti più 
vuol dire volare a vista de pájaro sull'intera poliedrica e ricchissima produzione zambraniana. Vuol dire, in sintesi, cogliere l'occasione per capirne la sua ragion d'essere più profonda.

Per entrare nel vivo del tema, vorrei sottolineare che María Zambrano gode di un ruolo centrale, nel lavoro svolto e da svolgere, per esaminare la recezione della mistica e le caratteristiche del pensiero mistico moderno nel xx secolo 5 . Questo perché la nascita e la crescita della sua "ragione poetica", fanno sì che la filosofa possa mettere in atto una modalità filosofico-poetica di scrivere, senza prescindere da un costante riferimento al más alláb, un'alterità rispetto all'umano che rimane un qualcosa di intellettualmente difficile da cogliere. I suoi testi ci lasciano molti paralleli con il pensiero mistico, tanto concettuali quanto linguistici che scopriremo progressivamente.

Sono ba+stati piccoli cenni introduttivi per mettere a nudo la radice mistica del pensiero di Zambrano e per preparare l'insieme di riflessioni che ci si propone di condividere.

\section{L' eredità di San Giovanni della Croce}

[San Juan de la Cruz] El santo de una ciudad castellana, temblorosa y ardiente, el santo de una antiquísima religión cuyo nombre es ya la poesía, el santo que es poeta.

María Zambrano, "San Juan de La Cruz (De la "noche oscura" a la más clara mística)"

María Zambrano ha il merito di aver conciliato il sapere classico antico della filosofia platonica con la più libera esperienza mistica espressa da San Giovanni della Croce. Credeva che il discepolo di Socrate, con la sua famosa dottrina sull'amore, avesse posto le basi dell'unità tra l'uomo e il divino nella cultura occidentale. A partire dall'Iperuranio, soprattutto dall'Idea del Bene, Platone aveva lasciato spa-

dettagliati del pensiero zambraniano grazie al confronto con la produzione teresiana. Le opere che ci consentiranno di ricostruire il confronto con questi autori vanno dalla produzione giovanile alla maturità, favorendo un'analisi di ampio spettro sull'evoluzione del pensiero zambraniano.

${ }_{5}$ Al riguardo, Llera, L., La razón humilde. Maria Zambrano y la tradición mistica española, Universidad Autónoma de Madrid Departamento de Antropología Social y Pensamiento Filosófico Español, Cuadernos Exilios, 2009 e Revilla Guzmán, C., "María Zambrano e la mistica di Castiglia", in B@belonline. Rivista online di Filosofia. Filosofia e mistica, a cura di Francesca Brezzi e Maria Teresa Russo, Roma TrE-Press, n. 1-2 (2016), pp. 131-144.

${ }^{6}$ Bundgaard, A., Más allá de la filosofía. Sobre el pensamiento filosófico-místico de María Zambrano, Madrid, Trotta, 2000 . 
zio a una connessione tra l'uomo e l'universo della conoscenza. Infatti, se l'amore è inteso come unità del sapere, il piano corporeo o la carne non sono necessariamente in conflitto con il regno delle idee - dove troverebbe dimora il divino-. Inserendosi su questa linea argomentativa, Zambrano comprende che si incorre in un errore metodologico e di contenuto se si opera una divisione tra il piano del corpo e il piano dell'anima. Tale divisione, contrariamente a quanto di solito si spiega nei manuali di storia della filosofia, non è l'unica tesi difesa dall'autore del Fedro; perché in realtà l'Amore platonico, che per la pensatrice spagnola "ya es mística", sopravvive ed è sostenuto dall'amore fisico, l'amore della carne. Sempre seguendo la scia di questa chiave interpretativa della storia del pensiero, il neoplatonismo del Medioevo e del Rinascimento sarebbero quindi due momenti storici in cui questa concezione dell'amore finisce per essere salvata, come un "categoría intelectual y social"'; a tal punto da persistere vigente nonostante l'ascetismo cristiano, sotto forma di poesia:

Éste es, creemos, el fundamento de toda mística: que el amor que nace en la carne (todo amor "primero" es carnal) tiene, para lograrse, que desprenderse de la vida, tiene también que convertirse, como decía Platón era menester realizar con el conocimiento. Y esta conversión, en verdad, se ha verificado por la poesía, en la poesía ${ }^{8}$.

L'amore si trasmette e continua a concretizzarsi grazie alla parola, a un logos verbalizzato in poesia; che a sua volta diventa la via d'accesso al sapere mistico, ne diventa la sua autentica base. In questo processo non viene a mancare una componente razionale nella misura in cui categorie e concetti possono essere utilizzati; purché si tenga presente che hanno un carattere mediatore, di congiunzione, tra l'uomo e il sacro. Assistiamo a un'ascesa naturale dalla dimensione materica al divino. In altre parole assistiamo a un percorso, questo è l'amore, che conduce l'essere umano a tali altezze epistemologiche, ed è in senso stretto un processo di conoscenza di se stessi, consentito dalla e grazie alla poesia.

A questo proposito, il professore Mariano Rodríguez ha sottolineato un paragone quanto mai chiarificatore riguardo il concetto di "salvezza dell'amante", che Zambrano ha scovato nella tradizione sufi e che ha conservato nel suo libro Filosofia ypoesía.

Il diavolo, secondo questa tradizione mistica araba, doveva sopportare la condanna di innamorarsi degli enti che il fiume del tempo portava verso la morte e, di fronte a questo incedere inesorabile, incapace di trattenere l'oggetto del suo amore,

\footnotetext{
7 Zambrano, M., Filosofia y poesía, in Obras Completas, Tomo I, Barcelona, Gutenberg, p. 732.

8 Ibid., p. 734.
} 
non poteva fare altro che piangere. Lo stesso accade al poeta, come Anacreonte ad esempio, autore di versi profondi e devastanti scritti per dar sfogo al dolore dovuto alla perdita della bellezza dei suoi amanti. Il poeta, l'amante, il demone sufi, sono tutti facce di uno stesso tipo antropologico ed epistemologico, che non può schivare il lamento e la sofferenza di un amore a cui deve sottomettersi dialetticamente? La via di salvezza di questo poeta-amante-diavolo risiede nell'amore platonico che gli permette di salvare se stesso e le cose amate. Questo iter di salvezza si consuma sotto la luce chiaroscurale di un logos che non definirei "oscuro", ma piuttosto una sombra llameante ${ }^{10}$ che permette di rendere eterno il momento corporeo, di ascendere alla maniera dei mistici.

Perché allora si percepiscono tanto orrore e pavore nutriti nei confronti del corpo nella tradizione occidentale e soprattutto nella cultura spagnola? La filosofa andalusa attribuisce al patrimonio paolino la promozione di questo disprezzo nei confronti della carne e delle passioni, consolidato nel faticoso tentativo di arginare l'ostacolo della tradizione ascetico-mistica cristiana che aveva imparato a convivere con il sapere greco ${ }^{11}$.

San Paolo, da questo punto di vista, avrebbe portato agli estremi la tesi platoni$\mathrm{ca}$, forse in parte fraintesa, del corpo come prigione o tomba dell'anima. La ragione poetica zambraniana ha tentanto, invece, di far convivere i due elementi e lo ha fatto riconoscendo nel misticismo castigliano un grande teatro di confronto e dialogo con il bagaglio filosofico di stampo platonico.

La pensatrice malagueña ha abbracciato la poesia di Juan de Yepes, Fray Juan de Santo Matías e infine San Juan de la Cruz, per il suo ruolo di recupero della concezione platonica dell'amore. Effettivamente, come ha giustamente posto sotto i riflettori il professore Rodríguez, uno dei punti chiave di Filosofía y poesía è l'identificazione del mistico castigliano come il restauratore più abile della poesia platonica e umana: " $\mathrm{OH}$ cristalina fuente/si en esos tus semblantes plateados/formases de repente los ojos deseados/que tengo en mis entrañas dibujados!" ${ }^{12}$. Nella sua spiegazione, il professore Rodríguez contestualizza questa presa di posizione zambraniana in due direzioni: 1)

\footnotetext{
9 Rodríguez, M., “Don Juan y San Juan: María Zambrano en 1939”, en Aurora, n. 15 (2014), p. 81.

Per un'analisi approfondita e rigorosa su Platone, San Juan de la Cruz e María Zambrano si consiglia: Langella, Simona; "The Poetic logos and the philosophical logos in María Zambrano", in Gant, Mark (Ed.), Revisiting Centres and Peripheries in Iberian Studies, Newcastle upon Tyne, Cambridge Scholars Publishing, 2019, pp. 177-188. La professoressa Langella è un'autrice di riferimento sul rapporto tra filosofia e mistica soprattutto del mondo ispanico, dal Siglo de Oro all'epoca contemporanea.

${ }^{10}$ Alludendo alla scrittrice e amica di Zambrano: Janés, C., Desde la sombra llameante, Madrid, Siruela, 2011.

11 Zambrano, M., Filosofía y poesía, op. cit., p. 715.

12 Rodríguez, M., op. cit., p. 81. Il verso di San Giovanni della Croce citato da Doña María in Filosofía y Poesía appartiene al Cántico Espiritual, Canto XII tra l'anima e il suo sposo.
} 
la sua critica tagliente contro il razionalismo, tessuta dal 1934 in Hacia un saber sobre el alma e 2) la sua dolorosa e personale esperienza dalla guerra civile, che la porterà ad alimentare una ragione poetica più misericordiosa durante il suo esilio ${ }^{13}$.

Grazie a questi studi, possiamo capire più precisamente l'enfasi che Doña María ha posto nel valorizzare la tradizione mistica castigliana, per poi collocarla nell'orbita della filosofia della sua ragione poetica che ha continuato ad elaborare nello svolgersi della sua lunga traiettoria esistenziale da esule, nonostante avesse già iniziato a portarla alla luce sin dalla sua giovinezza. Filosofía y poesía nasce in Messico, patria d'accoglienza in cui l'intellettuale iberica tenne una serie di conferenze nel 1939, confluite in un secondo momento in Pensamiento y poesía en la vida española. Leggiamo in questi testi una sua analisi della cultura europea, accompagnata da una sensibile ricostruzione e revisione della storia stessa della Spagna.

Il problema che viene presentato ai lettori, anche in La reforma del entendimiento español, è quello della costruzione della Spagna come dogma nazionale, con un cattolicesimo intransigente che promuoveva confessionalmente e che utilizzava il sacro per difendere l'impianto dell'unità nazionale. Tale Spagna inquisitoriale si trasformò in un dogma di fede, generando il "propio aniquilamiento de la fe" ${ }^{14}$. Oltre la critica a tale cultura ufficiale imposta, Zambrano ritorna alle radici della sua ricerca dell'amore platonico e del suo impegno per la custodia dell'armonia tra corpo e anima. María riteneva che vi fossero tre momenti storici in cui la cultura spagnola puntava verso questi propositi: la tradizione picaresca, incarnata nella figura di don Juan - ricerca dell'amore umano e esperienza radicale del presente ${ }^{15}$-; Seneca e la sua produzione filosofica o stoicismo, come pensiero laico di resistenza alle avversità $\mathrm{e}$, per concludere, ciò che ci interessa in dettaglio in questo frangente: il misticismo.

In particolare, nell'ottica zambraniana la mistica castigliana assume il ruolo di possibile salvatrice degli ideali platonici, dell'aspirazione antropologica a coniugare la libertà personale e il rispetto per l'altro, per gli altri. Il misticismo castigliano si oppone al misticismo tedesco, fondamentalmente per la "soledad absoluta del hombre frente a la tiránica voluntad divina"; perché non accoglie solo l'immagine di essere umani che si incamminano verso la morte quali naufraghi sconvolti nell'oceano della loro stessa esistenza. La mistica castigliana si distingue per la sua misericordia, considerando in armonica interconnessione, la meraviglia delle creature e del mondo che le circonda ${ }^{16}$.

\footnotetext{
13 Rodríguez, M., op. cit., pp. 82-84.

14 Zambrano, M., "La reforma del entendimiento español”, in Obras Completas, Tomo I, op. cit., p. 211.

${ }_{15}$ Per approfondire la lettura zambraniana del donjuanismo, si consiglia: Maillard, M. L., María Zambrano: la literatura como conocimiento y participación, Lleida, Ensayos/Scriptura, 1997, pp. 125-128.

${ }_{16}$ Zambrano, M., Pensamiento y Poesía en la vida española, in Obras Completas, Tomo I, op. cit., p. 585.
} 
Il caso di San Juan de la Cruz è per Zambrano una viva fonte di ispirazione, in un cammino che intraprende "dalla notte oscura alla mistica più limpida". Finalmente in una miriade di santi filosofi e scrittori, era riuscita a far la conoscenza di un santo poeta. Quando impiega questo aggettivo, bisogna tenere presente che non si riferisce esclusivamente ai versi, al genere letterario noto come poesia, in contrapposizione alla prosa o al dramma. La filosofa veleńa concepisce la poesia nel suo senso originario, ovvero come $\pi$ oinoıs, come creazione; in pieno legame diretto con la vita, con il sapere, con l'anima e con il corpo. Questo spiega perché quando si interroga in questi termini: “QQué religión es ésta del Carmen que permite la poesía, que la engendra?” ${ }^{17}$ include, nella stessa domanda, la descrizione del suo operato. Dunque, come ammette lei stessa, anche San Giovanni della Croce ha scritto in prosa, ma sempre con il fine ultimo di far luce sul contenuto della sua poesia. Con i suoi versi lui fa risplendere la città castigliana da cui proviene la sua stessa poesia, le dà vita. La città con i suoi elementi, il suo canto, la sua terra, i suoi abitanti. È la poesia che illumina la solitudine della Castilla di San Juan, della sua Spagna, della stessa Zambrano.

María sente un parallelismo intrinseco tra l'ascesa della poesia di San Giovanni della Croce e il suo viaggio mistico. Allo stesso tempo, trova in questo legame profondo la cultura spagnola, l'orizzonte da cui guardarla. Si prospetta un'ascesa, la "subida al monte", attraverso cui San Giovanni vede passare l'esistenza umana. È un cammino arduo, ascetico perché deve purificare l'anima; ma senza distruggerla o danneggiarla. Riconosce in San Juan la proiezione della sua concezione di misticismo, come qualcosa di naturale, umano, perché "la mística es algo que sucede dentro del alma" 18 , anche se in seguito si combina con qualcosa di innaturale, cioè ciò che è al di fuori di essa; ma sempre in un contesto intelligibile e intelligente:

En realidad, lo que sucede en la mística no es en manera ajeno a lo humano, ni es cosa de impostores, ni dementes, como el positivismo creyera. Y por extrańa que se suponga a la mística dentro del género humano, su gran corriente, tan fecunda e inextinguible, es como para hacer meditar. Para hacer meditar y pensar que lo que sucede en la mística está al menos fundado en la naturaleza humana, en una posibilidad esencial a ella, tal vez en una condición que se revela en la mística más que en cosa alguna ${ }^{19}$.

Allo stesso tempo non si fa meno acuta la critica al razionalismo, in questo caso nella sua versione positivista. La figlia di Don Blas Zambrano fa appello a una metafora tratta dall'ambito della biologia per mostrare precisamente l'essenza

\footnotetext{
17 Zambrano, M., "San Juan de la Cruz de la noche obscura a la más clara mistica", in Obras Completas, Tomo I, op. cit., p. 285

18 Ibid., p. 287.

19 Ibid.
} 
e la natura del misticismo. Il mistico in senso sanjuanista è come una crisalide che mangia (autofagia) il proprio bozzolo per uscire da se stessa; il che significa istinto di sopravvivenza, ma oltre a questo, come dice Zambrano, si tratta della sua "sed de la vida" dovuta all'amore, un desiderio ineludibile di esistere e di avere una "figura" ${ }^{20}$. Sebbene annientando il suo involucro, l'anima si ferisce, rimane esposta in un nulla che è solo temporaneo; un instante in cui si apre totalmente all'altro, a Dio; un'alterità che non osa conoscere, ma in cui vuole essere. L'unità di conoscenza di cui si è parlato prima non ha un senso epistemologico, ma ontologico. Il mistico prova una forte esperienza di alterità, di vuoto e di deserto, ma si inoltra ben oltre. Questo passaggio è il vero inizio, la fonte della creazione. Per María Zambrano, il misticismo di San Juan de la Cruz è un misticismo della creazione, perché non anela il semplice possesso di una figura, ma è totale proiezione verso l'altro con una veemenza che corrisponde a un amore ben preciso, l'amore platonico.

L'amore in San Juan de la Cruz lo traduce simbolicamente in una fiamma, così affine alla sua visione dell'aurora come principio dell'atto creativo, fonte dell'etica, che viene poi superato per arrendersi alla fame d'amore che il mistico vive. È una visione in cui si varca la soglia della sfera psichica e morale, della morte senza abbandonare completamente la realtà: al contrario, ci si immerge di più in essa, nelle cose, si raggiunge uno stato di piena connessione con tutto il creato. È una mistica "clara" che permette l'articolazione di poesia, religione e pensiero in un'unione armoniosa, un' unione perfetta come afferma Zambrano, di amore e conoscenza.

L'anima si era persa su quel sentiero oscuro per incontrarsi, non con il desiderio, ma con l'amore e la sua fonte, che non viene distrutta; in questi termini si dà la sua trascendenza. Ecco perché María Zambrano amava molto i versi: “¡Oh cristalina fuente/si en estos tus semblantes plateado/ formases de repente/los ojos deseados/ que tengo en mis entrañas dibujados". Lì, indagando, ha trovato l'amore come oggettività, che dirige e non si esaurisce, che viene addirittura ricreato nella distruzione, che è. Scova in San Giovanni della Croce la massima e completa commistione tra misticismo e poesia nella creazione; un sapere che va oltre la conoscenza scientifica, pur aiutandola a formare i suoi concetti. L'amore è la base della conoscenza:

Sin la exigencia del amor, la mente no habría llegado a forjar la idea, la posesión de la presencia y la figura tal como ella puede únicamente alcanzarse. La objetividad no es posible sin el amor, y por parte del hombre, quizá no sea más ${ }^{21}$.

\footnotetext{
${ }^{20}$ Ibid., pp. 288-289.
}

${ }^{21}$ Ibid., p. 294. 
Questa riflessione l'ha condotta fino all'origine della filosofia stessa, perché se l'amore si manifesta con tutta la sua forza nella poesia e nel misticismo, qual è il suo ruolo nella filosofia? Pochi filosofi, da questo punto di vista, hanno eguagliato il percorso intrapreso da San Juan. Chi si è avvicinato - pur se in modo razionale- è stato Spinoza, presente anche nelle riflessioni dottorali zambraniane come "ebreo di origine spagnola”. Mentre San Giovanni trovava la vita nella poesia, Spinoza proteggeva la vita e la sua visione della divinità sotto il velo della ragione. Questi due autori cercavano l'amore in due modi diversi, raggiungendo la medesima desiderata unità di conoscenza e oggettività; di cui Doña María sentiva la mancanza nella sua terra e nel suo tempo: “¿Por qué, Señor san Juan, no recobra Castilla su objetividad?" 22 .

In questo saggio su "San Juan de la Cruz, de la 'noche oscura' a la más clara mística", la filosofa andalusa paragona il mistico castigliano al teologo spagnolo Miguel de Molinos, vissuto a Roma e condannato dall'Inquisizione. Autore di un'importante Guida spirituale, Molinos ha incarnato in maniera esemplare la tendenza quietista. Zambrano oppone al misticismo creativo del padre della Noche oscura, quella che considerava in questo momento una mistica che si concludeva nel momento della distruzione, nel silenzio, nel nulla e non proseguiva l'ascesa al Monte Carmelo. Per Molinos, allora, la presenza e la vita si estinguerebbero fino a toccare la quiete e non ci sarebbe più niente da aggiungere a questo processo da consumare.

Tuttavia, la stessa autrice rivedendo il suo testo, annoterà a piè pagina, anni dopo, una versione rivisitata della mistica di Molinos e la sua nozione di nada. Il risultato di questo cambio di prospettiva comparirà nel suo articolo "Miguel de Molinos recuperado", firmato a La Pièce nel 1974 e pubblicato nel numero 338 della rivista Insula del 1975. In realtà, si tratta di una recensione critica dell'edizione della Guida spirituale del poeta José Ángel Valente, che comprendeva un saggio di interpretazione e un testo recuperato intitolato Defensa de la contemplación. Dopo trecento anni, la Guía espiritual tornava ad apparire in spagnolo. In un ambiente che si stava avvicinando alla transizione democratica, María legge Molinos sotto una lente d'osservazione diversa: la rivalutazione del misticismo nel contesto del pensiero filosofico europeo e spagnolo.

Rivisitando Molinos, Zambrano interpreta in modo differente la stessa storia europea. Riscopre un Molinos che non è assorto nel nulla, bensì un autore che nella propria esistenza trova un punto d'accordo tra la chiarezza della luce e la profondità della vita, attraverso il suono del linguaggio; anche quello del silenzio. L'oscurità si accende al ritmo di una musica soffocata. Mentre il suo corpo si stava deperendo

22 Ibid., p. 297. 
sepolto vivo, non si stava dirigendo solo verso il nulla; nella sua vita si stava accendendo anche un'alba inestinguibile ${ }^{23}$.

Per concludere, ricordiamo che nel discorso che ha preparato in vista della consegna del Premio Cervantes nel 1988 -è stata la prima donna a vincerlo-, Zambrano ha paragonato l'autore del Don Chisciotte, colto in punto di morte, a San Giovanni della Croce. Del Cantico spirituale l'intellettuale di Malaga apprezza la ricerca dell'amato invisibile, la sua assenza e la ricerca delle sue orme. L'essere umano è sempre in attesa di stanare la presenza dell'amato, anche se la natura muta costantemente. Dopo tutto, questo è ciò che è accaduto a Cervantes, a San Juan e alla stessa María Zambrano; che, di ritorno in una Spagna di nuovo democratica, ha affermato:

voy a intentar seguir buscando la palabra perdida, la palabra única, secreto del amor divino-humano. La palabra tal vez señalada por aquellas otras palabras privilegiadas, escasamente audibles, casi como murmullo de paloma:

Diréis que me he perdido,

Que, andando enamorada,

Me hice perdidiza y fui ganada ${ }^{24}$

\section{L'eredità di Santa Teresa}

"Vivo ya fuera de mí", decía Santa Teresa, y no es nada específico de ella. Vivir fuera de sí, por estar más allá de sí mismo. Vivir dispuesto al vuelo, presto a cualquier partida. Es el futuro inimaginable, el inal-

\footnotetext{
${ }^{23} \mathrm{Si}$ è seguito il recupero del testo originale presso l'archivio della Fondazione María Zambrano edito da Serantes, M. A., "María Zambrano en Miguel de Molinos reaparecido: un estudio crítico", in Rudesindus, n.7 (2011), pp. 292-293. María Zambrano aveva già condiviso un’opinione differente su Molinos nel saggio Resignación y esperanza, paragonando la Guida Spirituale all'Etica di Spinoza e alla famosa poesia di incerta paternità "No me mueve mi Dios para quererte", che alcuni attribuiscono a Santa Teresa, mentre Zambrano ritiene che il suo legittimo autore sia San Juan de la Cruz. Zambrano, M. Pensamiento y poesía en la vida española, in Obras Completas, Tomo I, op. cit., p. 644.

${ }^{24}$ Zambrano, M., "Discurso en la entrega del Premio Cervantes 1988”, en A.A.V.V., Premios Cervantes. Una literatura en dos continentes, Valladolid, Ministerio de Cultura, 1994, pp. 262-263.

Il discorso è stato letto dall'attrice Berta Riaza, su richiesta della stessa María. I versi del Cantico spirituale di San Juan de la Cruz e la sua riflessione sono stati pubblicati in un primo momento in Claros del Bosque, dato che l'autrice ha rielaborato il suo Discorso del 1988 con il materiale che aveva scritto in precedenza. A questo proposito, risulta molto interessante il richiamo zambraniano al celebre passo dantesco: "Nel mezzo del cammin di nostra vita/mi ritrovai per una selva oscura/che la diritta via era smarrita". Il poeta fiorentino ci parla della sua interiorità, come fece san Giovanni: "Se hizo perdidiza el alma esposa de San Juan de la Cruz en medio del camino estrecho de la subida al Monte paso a paso en una búsqueda metódica - un método el suyo de rigor intelectual, más implacable que ninguno de los propiamente filosóficos”. Zambrano, M., Dante, specchio umano. A cura e con un saggio introduttivo di Elena Laurenzi, testo spagnolo a fronte, Troina, Città Aperta, 2007, p. 114.
} 
canzable futuro de esa promesa de vida verdadera que el amor insinúa en quien lo siente. El futuro que inspira, que consuela el presente haciendo descreer de él; que recogerá todos los sueños y las esperanzas, de donde brota la creación, lo no previsto. Es la libertad sin arbitrariedades. El que atrae el devenir de la historia que corre en su busca. Lo que no conocemos y nos llama a conocer. Ese fuego sin fin que alienta el secreto de toda vida. Lo que unifica con el vuelo de su trascender vida y muerte, como simples momentos de amor que renace siempre de sí mismo. Lo más escondido del abismo de la divinidad; lo inaccesible que desciende a toda hora.

Zambrano, M., El hombre y lo divino

Secondo il poeta César Antonio Molina, "María [Zambrano] se extasiaba en Santa Maria della Vittoria, dentro de la capilla Cornaro, en el Quirinal, en torno a las Quattro Fontane" ${ }^{25}$. In questa cappella barocca di una chiesa romana dei Carmelitani Scalzi, Gian Lorenzo Bernini ha immortalato il momento della transverberazione di Santa Teresa ${ }^{26}$, visione estatica che ha fortemente influenzato María Zambrano. Attira l'attenzione che la filosofa spagnola faccia poco riferimento a questo dettaglio primario nei suoi testi, in paragone all'inchiostro versato parlando di San Juan de la Cruz, Miguel de Molinos o Giordano Bruno.

Per Zambrano, Santa Teresa ha dialogato senza paura con la morte e, in egual misura, si è rallegrata della vita, in piena corporeità. La vita le era "presente siem-

${ }^{25}$ Molina, C. A., "María Zambrano, paseos por Roma”, in A.A.V.V., María Zambrano en su centenario: los años de Roma (1953-1964), Roma, Instituto Cervantes, 2004, disponibile online: https://cvc.cervantes.es/literatura/ zambrano_roma/molina.htm.

${ }^{26}$ La Transverberazione di Santa Teresa d'Avila, più comunemente conosciuta come l'Estasi di Santa Teresa, è una scultura in bronzo dorato e marmo di Gian Lorenzo Bernini, realizzata tra il 1647 e il 1652 . L’opera si trova nella cappella Cornaro presso la chiesa di Santa Maria della Vittoria, a Roma. Si tratta di uno dei massimi esempi del baracco romano, al quale è possibile aggiungere un secondo esempio della produzione più matura dello scultore. Conservata presso San Francesco a Ripa a Roma, l'Estasi della beata Ludovica Albertoni, datata 1674, arricchisce infatti, un'analisi sul misticismo italo-iberico che può essere elaborata a partire dal punto di vista dell'arte. Nella sua ineguagliabile ricchezza artistica, Roma offre un'ulteriore ed interessantissimo spunto di riflessione sul misticismo. A Trastevere, presso la basilica di Santa Cecilia, ai piedi dell'altare maggiore notiamo una statua che raffigura la santa patrona della musica di Stefano Maderno, risalente al 1599. Una copia fedele dell'opera si può ammirare anche presso la Reale Accademia di Spagna a Roma. La santa è colta nel momento in cui, secondo la leggenda, si prepara a raggiungere l'al di là, in un'agonia durata tre giorni, cantando a Dio, in unione con Dio. Quando il corpo fu ritrovato nelle catacombe di S. Callisto sulla via Appia, la santa avvolta in un saio bianco e dorato simbolo di purezza, aveva lo sguardo ritratto alla vista altrui per mostrare i segni del martirio e le mani poste in modo tale da indicare il numero tre, la Trinità. Stiamo parlando di una figura femminile che la "ragione poetica" zambraniana potrebbe analizzare con i suoi strumenti ermeneutici. In effetti, Santa Cecilia è una figura femminile mediatrice tra paganesimo e cristianesimo, vita e morte, attenta a sfidare le gerarchie sociali del suo tempo, superando i limiti tra piano terreno e sacro grazie alla sua esperienza mistica e musicale. Inoltre, la figura ci permetterebbe di analizzare il ruolo della musica nel pensiero zambraniano, così legato al divino in quanto paradigma di espressione del razionalmente incomunicabile. Ci limitiamo a sottolineare veloci spunti di riflessione su cui è opportuno continuare ad indagare più a fondo. 
pre" e vi si riferiva sempre, come esistenza ${ }^{27}$. Santa Teresa, in questo senso, considerava materna la "materia stessa"; la carne e il suo "palpitar" ${ }^{28}$. Nel corso della storia spagnola, in un mondo in cui le donne erano il pilastro della vita domestica e della famiglia; Santa Teresa ha incarnato lo "spirito" che camminava tra le stoviglie, "el pobre espiritu - dice Zambrano- que ennoblece tanto a los cacharros y que a veces desfallece entre ellos, sin que nadie le auxilie" 29 .

La lettura zambraniana di Santa Teresa differisce necessariamente da quella di San Juan de la Cruz per le caratteristiche peculiari di ognuno dei due autori. Mentre la discepola di Ortega in San Juan ha indivituato e studiato un itinerario di interiorità, che muove dall'assenza e dall'annullamento del sé, verso la contemplazione e la creazione; in Santa Teresa si confronta con un modello spirituale che osserva con una punta forse superiore di ammirazione. La totale esperienza estatica teresiana, il suo uscire da sé stessa pur continuando ad aggirarsi nei meandri di una mistica della carne, molto vicina al cuore umano, fanno sì che la filosofa si avvicini alla santa abulense con livelli di cautela particolarmente accentuati. Zambrano arriva a queste conclusioni ascoltando la voce di Santa Teresa, in Las Moradas: "el alma está fuera de sí para querer entrar dentro de sî" ${ }^{30}$. La sua esperienza di estasi non la vede come un'esperienza innaturale, ma come un ponte con la più profonda intimità della natura umana, con il cuore. Platone e i primi neoplatonici, come Plotino, seguono le tracce del cuore; ed è anche grazie a loro che Zambrano schiude quell'esperienza di uscire da sé stessa ben presente nel suo concetto di "alma pura" ${ }^{1}$. Il grande testo che gli studiosi descrivino quale opera più specificamente di natura mistica di María Zambrano è El hombre y lo divino; dove l'autrice si concentra molto di più sulla filosofia greca che sulla stessa tradizione cristiana. I cieli o la conoscenza dei corpi celesti corrispondono per i Greci, secondo María, a un dialogo intimo dell'anima che si estende fuori di sé, nello sforzo di gioire della sua interiorità; senza perdersi.

Da questo punto di vista, ci sarebbe già un'esperienza di estasi nella nascita della matematica, una nozione metafisica che, insieme alla musica, ha partecipato alla risonanza dei corpi celesti. Questa è la saggezza greca che aspirava all'assoluto, a una "conoscenza pura" come la definisce Doña María, che corrisponde a una sospensione del tempo, a un atto che inizia con gli occhi, la visione, e che

\footnotetext{
27 Zambrano, M., "San Juan de la cruz: de la noche obscura a la más clara mística", op. cit., p. 287.

28 Zambrano, M., Pensamiento y poesía en la vida española, op. cit., p. 585.

29 Ibid., p. 646.

${ }^{30}$ Zambrano, M., Hacia un saber sobre el alma, Madrid, Alianza, 2000, p. 96.

31 Zambrano, M., El hombre y lo divino, México, FCE, 2012, p. 100.
} 
si trasforma in amore una volta che l'anima esce da sé stessa. Per questo non si risparmia la critica contro la filosofia moderna, per aver ridotto l'amore di questo sguardo verso il celeste, al solo regno delle passioni. Ebbene, per lei le passioni, giustamente comprese, sono gli ingredienti necessari che si combinano nell'unione di conoscenza e amore.

In cosa consiste l'amore umano? A questo punto per rispondere, Zambrano incontra Sant'Agostino, un magistrale autore neoplatonico; che afferma che il peso dell'uomo, il suo centro gravitazionale, è l'amore: "Il mio peso è il mio amore" ${ }^{2}$. María interpreta alla luce di questa tradizione, la sua visione filosofica dell'uscire da se stessi: ovvero vivere fuori da sé, per e verso la persona amata. Ecco che può comprendere allora la mistica teresiana nella sua complessità. Vivere al di fuori di se stessi è una condizione umana, uno stato particolare in cui la persona si assume dei rischi ontologici di primo ordine, proiettandosi nella promessa del vero amore afferrabile nella sua vita futura. È un fuoco che la spinge, la ispira, fino a riconoscere anche in Santa Teresa il profilo di una mistica della creazione. Si dispiega un volo di libertà che condensa non solo la conoscenza, ma anche ciò che ignoriamo e desideriamo sapere, ciò che cerchiamo ${ }^{33}$.

In contrasto con San Juan de la Cruz, secondo Zambrano, Santa Teresa non teme la morte o delle sue espressioni, perché nel suo programma mistico è capace di trascenderle nell'amore senza tempo, sul piano dell'eternità. Si affaccia, dunque di nuovo, nelle riflessioni zambraniane, un legame indissolubile tra filosofia greca e misticismo spagnolo. María crede che il meglio delle due tradizioni sia una base spirituale condivisa che arricchisce l'animo umano e gli permette di attingere alla sua più profonda e autentica identità nella sua interiotà $\mathrm{e}$ in un costante percorso di riconoscimento con l'alterità:

"Luz de luz" es la fórmula más alta de la Teología que expresa el punto de identidad entre la Filosofía griega y la Fe cristiana. En la luz coincidieron pensamiento y religión cristiana. Religión de luz viva y actuante en todos los anhelos e intentos; en las esperanzas y en las creaciones más dispares y aun contrarias, pues la divergencia de credos estéticos nunca llegó hasta este inquietante periodo en que se deshacen las formas y el rostro humano se oculta. Eclipse de lo humano que se verifica en la vida también, es la noche oscura de lo humano que semeja un retiro de una luz y un logos donde no se encontraban ya sino diferencias, discernimientos; una retirada y un retroceso del Dios de la teología en busca del Dios que devora y quiere ser devorado ${ }^{34}$.

\footnotetext{
32 Sant'agostino, Confessioni, 1Ibid. 13, cap. 7, Milano, Editrice Bibliografica, edizione di Giuliano Vigini, 1993, p. 393.

33 Zambrano, M., El hombre y lo divino, op. cit., p. 276.

34 Zambrano, M., La destrucción de las formas, Bogotá, Universidad Nacional de Colombia, 2004, p. 23.
} 
La notte oscura dell'umano, di memoria sanjuanista, è solamente una fase del percorso ascendente verso la luce. Entrambi i mistici castigliani tendono allo stesso obiettivo.

Ma, mentre in San Juan de la Cruz emerge l'influenza lampante del platonismo, in Santa Teresa è più evidente la presenza di Sant'Agostino, neoplatonico e cristiano. Alla fine, entrambe le strade si incrociano e addirittura si intersecano con le virtù degli stoici, in particolar modo con la loro impassibilità (non soffrono il passare del tempo, né l'odio accumulato nei secoli).

Santa Teresa è per Zambrano un esempio paradigmatico del raggiungimento della moltiplicazione dei tempi, del vivere contemporaneamente tempi diversi, perché non ne sente il peso e li supera. Se le è possibile un lavoro così prodigioso è solo grazie all'estasi, con cui sopravvive nell'instante temporale umano e supera le ingiustizie di questo mondo. Il suo sprone è la forza dell'amore, che per la pensatrice andalusa è al di là della storia; difatti, non si può scrivere una storia dell'amore. La sua eternità risplende e trionfa in un evento intimo, interiore, nel silenzio di sé stessi. Ora non è più un mistero il nucleo fondante dell'esperienza teresiana dal punto vista zambraniano. Santa Teresa, è riuscita a vivere dentro e fuori di sé:

Pues los místicos lograron abstraer el tiempo casi eternamente, vivir en dos tiempos o en tres, como le sucedió a Teresa de Ávila, tan lejos que la tenía [la impasibilidad] y había vuelto a pensar en ella, ¿por qué? Quizá porque Teresa vivió el «instante» en el éxtasis, el tiempo histórico en su acción en el mundo, entre el mundo, y vivió también el tiempo de la meditación. Y a través de su "vida» sé veía claro lo que en la meditación hay de decadencia, de «a falta de otra cosa». Y en cuanto a la acción, el querer realizar o encontrar el equivalente del momento de éxtasis. Pues la acción, ahora descubría su atractivo, era una especie de «éxtasis», la acción verdadera. ${ }^{35}$

\section{Conclusioni}

María Zambrano è una pensatrice delle viscere, dell'interiorità e della carne, le stesse categorie che ha ereditato dall'esperienza teresiana. Come ha suggerito Fanny Rubio, questa grande esponente della Scuola di Madrid è d'accordo in questo senso anche con il poeta Luis Cernuda, per tutto ciò che concerne la sua descrizione dell'esperienza della vita; soprattutto per quanto riguarda la nozione

\footnotetext{
35 Zambrano, M., "La multiplicidad de los tiempos", in Anthropos, Antología, Selección de Textos, Barcelona, (marzo-abril 1987), p. 9
} 
di risveglio che influenza l'intera percezione della nostra soggettività a partire dall'alterità ${ }^{36}$.

È vero che Zambrano condivide anche un parallelismo contestuale con Santa Teresa, che Jenny Haase ha invitato a valorizzare molto bene: mentre la prima autrice ha subito la Guerra Civile, la Santa di Avila ha dovuto remare contro le acque della Controriforma ${ }^{37}$. Le loro esperienze storiche hanno esercitato pressione in modo notevole sulla loro concezione dell'anima, sulla loro più profonda antropologia, sulla cura del loro linguaggio e sulla dimensione didattica che davano alla loro interiorità. In accordo con questi elementi, ho potuto rielaborare e condividere il raffinato sposalizio di soggettività, alterità umana e alterità divina che in entrambe le autrici emerge a gran voce. Allontanandomi in parte dall'interpretazione che la professoressa Haase ha reso in chiave psicanalitica, ritengo che sia Zambrano che Santa Teresa investano nell'autorealizzazione della persona e che il loro modello relazionale tra soggettività e alterità, consenta un approfondimento sul giusto atteggiamento etico e politico da attuare legato ai concetti di pietà, carità, amore e misericordia. Se uniamo tutto questo all'importanza che San Juan ha ottenuto nel pensiero zambraniano per comprendere l'essenza della cultura spagnola e della sua "ragione poetica", capiamo quanto sia lecito affermare che il pensiero di Doña María sia una filosofia dalle radici profondamente mistiche.

È giunta l'ora di pensare alla celebre "Creazione di Adamo" 38 che il talento di Michelangelo ha potuto portare alla luce. Affidiamoci di nuovo al sostegno dell'arte.

Si tratta della raffigurazione del momento in cui Dio porge la sua mano verso quella umana. Ma non solo Dio anela il contatto, anche il desiderio di unione dell'uomo con il suo Creatore traspare dallo sforzo di approssimare il suo palmo a quello divino. Le due mani si sfiorano soltanto. Non si tratta semplicemente di un'assenza, una cavità priva di contenuto. È la prospettiva in cui sorge e cresce

\footnotetext{
36 Rubio, F., "María Zambrano y las formas de lo sagrado en la poesía española a partir de El hombre y lo divino", in A.A.V.V., María Zambrano en su centenario: los años de Roma, Roma, Instituto Cervantes, 2004, disponibile online: https://cvc.cervantes.es/literatura/zambrano_roma/rubio.htm. Citazione dal verso della poesia Lázaro: " "Alguien dijo palabras / de nuevo nacimiento. / Mas no hubo alli sangre materna / ni vientre fecundado / que crea con dolor nueva vida doliente (...) / Quise cerrar los ojos / buscar la vasta sombra / la tiniebla primaria / (...) Cuando un alma doliente en mis entrañas / gritó, por las oscuras galerías (...) / Entonces, hondos bajo una frente, vi unos ojos / llenos de compasión, y hallé temblando un alma donde mi alma se copiaba inmensa, / por el amor dueño del mundo»".

${ }^{37}$ Hasse, J., "María Zambrano y la mística. Leyendo a una filósofa moderna con Santa Teresa de fondo", in SymCity, n. 4, (2013), p. 12.

${ }^{38}$ Si ringrazia vivamente la professoressa Lucia Parente, per aver invitato alla riflessione sul valore filosofico del capolavoro michelangiolesco in: Parente, L., Una voce che veniva da lontano. Saggi e ricerche su María Zambrano, Milano, Mimesis, 2018, pp. 147-148.
} 
l'esperienza mistica di cui hanno osato parlare San Giovanni e Santa Teresa e di conseguenza, l'esperienza filosofica che hanno affidato a María Zambrano.

Quella frazione temporale in cui Dio e l'uomo non arrivano a toccarsi, affrescata in modo sublime dalle altissime doti artistiche delle maestranze fiorentine del Cinquecento, non è altro che la manifestazione dell'importanza immortale ed universale del desiderio umano di ricongiungersi con il sacro. Vediamo come il Creatore e la creatura sfidano la loro finitudine, senza annullarsi mutuamente. Tra la mano del Creatore e del creato, affondano le radici del carattere metafisico della filosofia, perché è lì che si dà la necessaria relazione tra i due protagonisti dell'esistenza e del pensiero, della vera libertà e dignità umane.

Abbiamo iniziato parlando delle pennellate di Tiziano, finiamo riflettendo su altre pennellate rinascimentali estratte non solo da un famosissimo affresco, ma da un intero percorso filosofico, che congiunge la grande mistica spagnola al cuore della produzione di María Zambrano.

Doña María è una filosofa che non ha mai smesso di unire pensiero e carne, piano divino e vita, dimensione del sacro e uomo, intravedendo un cammino che attraversando le pieghe della filosofia e la poesia, si è diretto verso un sapere sull'anima $^{39}$, o detto in termini più generali, verso un sapere sul sacro.

39 Il riferimento al libro Hacia un saber sobre el alma di María Zambrano è una scelta argomentativa a favore della tesi che si è tentato di difendere nel corso del presente testo. Per sottolineare come sin dalla nascita della "ragione poetica”, percepibile nel libro preso a riferimento, sia palpabile la traiettoria che la filosofia zambraniana sceglie in linea con la tradizione del misticismo spagnolo del xvi secolo. 


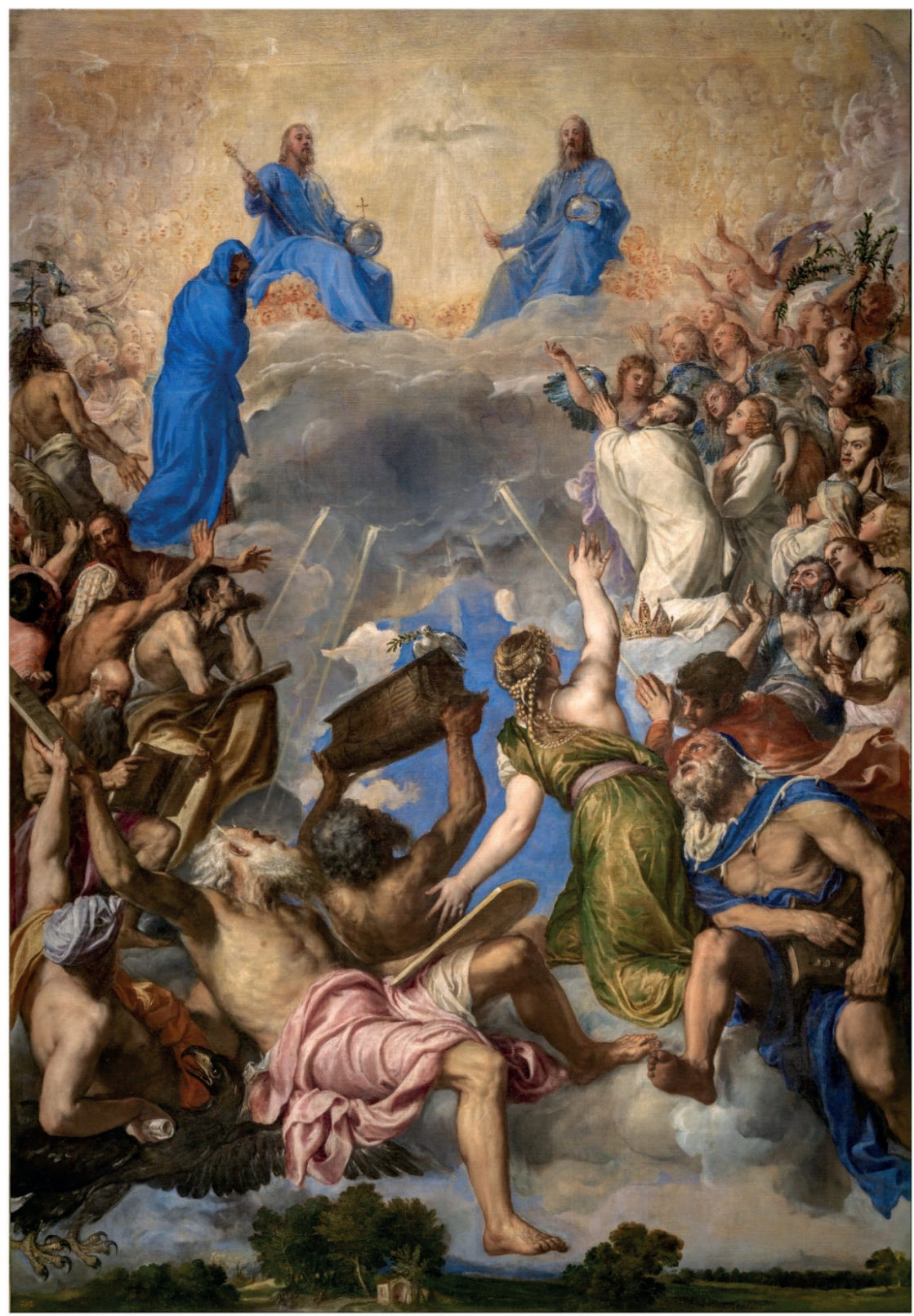

La Gloria, Tiziano, Vecellio di Gregorio. c. 1551-1554. Museo del Prado. Uso accademico senza fini di lucro. 


\section{REFERENCIAS BibLIOGRÁficas}

Bundgaard, A., Más allá de la filosofía. Sobre el pensamiento filosófico-místico de Maria Zambrano, Madrid, Trotta, 2000.

Croce, E., Due città, Milano, Adelphi, 2004.

Hasse, J., "María Zambrano y la mística. Leyendo a una filósofa moderna con Santa Teresa de fondo", in SymCity n. 4, (2013), pp. 1-13.

JanÉs, C., Desde la sombra llameante, Madrid, Siruela, 2011.

Langella, Simona; "The Poetic logos and the philosophical logos in María Zambrano", in Gant, Mark (Ed.), Revisiting Centres and Peripheries in Iberian Studies, Newcastle upon Tyne, Cambridge Scholars Publishing, 2019, pp. 177-188.

Llera, L., La razón humilde. María Zambrano y la tradición mistica española, Universidad Autónoma de Madrid, Departamento de Antropología Social y Pensamiento Filosófico Español, Cuadernos Exilios, 2009.

Maillard, M. L., María Zambrano: la literatura como conocimiento y participación, Lleida, Ensayos/Scriptura, 1997.

Molina, C. A., "María Zambrano, paseos por Roma", in A.A.V.V., María Zambrano en su centenario: los años de Roma (1953-1964), Roma, Instituto Cervantes, 2004, disponibile online: https://cvc.cervantes.es/literatura/zambrano_roma/ molina.htm

Parente, L., Una voce che veniva da lontano. Saggi e ricerche su María Zambrano, Milano, Mimesis, 2018.

Revilla Guzmán, C., "María Zambrano e la mistica di Castiglia", in B@belonline. Rivista online di Filosofia. Filosofia e mistica, a cura di Francesca Brezzi e Maria Teresa Russo, Roma TrE-Press, n. 1-2 (2016), pp. 131-144.

Rodríguez, M., “Don Juan y San Juan: María Zambrano en 1939”, in Aurora, n. 15 (2014), pp. 80-89.

Rossi, R., Juan de la Cruz. Silencio y creatividad, Traducción de Juan-Ramón Capella, Madrid, Minima Trotta, 2010.

Rossi, R., Teresa de Ávila. Biografía de una escritora, Madrid, Editorial Trotta, 2015. 
Rubio, F., "María Zambrano y las formas de lo sagrado en la poesía española a partir de El hombre y lo divino", in A.A.V.V., María Zambrano en su centenario: los años de Roma, Roma, Instituto Cervantes, 2004, disponibile online: https://cvc.cervantes.es/literatura/zambrano_roma/rubio.htm

Sant'agostino, Confessioni, lib. 13, cap. 7, Milano, Editrice Bibliografica, edizione di Giuliano Vigini, 1993.

Serantes, M. A., "María Zambrano en Miguel de Molinos reaparecido: un estudio crítico", in Rudesindus, n. 7 (2011), pp. 281-293.

Zambrano, M., "Discurso en la entrega del Premio Cervantes 1988", in A.A. V.V., Premios Cervantes. Una literatura en dos continentes, Valladolid, Ministerio de Cultura, 1994, pp. 257-263.

Zambrano, M., "La multiplicidad de los tiempos", in Anthropos, Antología, Selección de Textos, Barcelona, (marzo-abril 1987), pp. 7-11.

Zambrano, M., "La reforma del entendimiento español", in Obras Completas, Tomo I, Barcelona, Gutenberg, 2015, pp. 205-220.

Zambrano, M., "San Juan de la Cruz de la noche obscura a la más clara mística", in Obras Completas, Tomo I, Barcelona, Gutenberg, 2015, pp. 284-297.

Zambrano, M., Dante, specchio umano, a cura e con un saggio introduttivo di Elena Laurenzi, testo spagnolo a fronte, Troina, Città Aperta, 2007.

Zambrano, M., El hombre y lo divino, México, FCE, 2012.

Zambrano, M., Filosofía y poesía, in Obras Completas, Tomo I, Barcelona, Gutenberg, 2015, pp. 657-777.

Zambrano, M., Hacia un saber sobre el alma, Madrid, Alianza, 2000.

Zambrano, M., La destrucción de las formas, Bogotá, Universidad Nacional de Colombia, 2004.

Zambrano, M., Pensamiento y Poesía en la vida española, in Obras Completas, Tomo I, Barcelona, Gutenberg, 2015, pp. 553-656.

DOI: https://doi.org/10.15366/bp2020.25.012

Bajo Palabra. II Época. No25. Pgs: 251-272 
